\title{
Biodegradable Antimicrobial Agent/Analgesic/ Bone Morphogenetic Protein-Loaded Nanofibrous Fixators for Bone Fracture Repair
}

\author{
Yi-Hsun Yu iD \\ Yu-Ting Lin ${ }^{2}$ \\ Yung-Heng Hsu' \\ Ying-Chao Chou' \\ Steve WN Ueng (D) \\ Shih-Jung Liu (iD) ${ }^{1,2}$ \\ 'Department of Orthopedic Surgery, \\ Bone and Joint Research Center, Chang \\ Gung Memorial Hospital, Tao-Yuan, \\ 33305, Taiwan; ${ }^{2}$ Department of \\ Mechanical Engineering, Chang Gung \\ University, Tao-Yuan, 33302, Taiwan
}

Correspondence: Shih-Jung Liu Department of Mechanical Engineering, Chang Gung University, 259, Wen-Hwa

Ist Road, Kwei-Shan, Tao-Yuan, 333,

Taiwan

Tel +886-3-21I8I66

Fax +886-3-21।8558

Email shihjung@mail.cgu.edu.tw
Purpose: Postoperative infection and pain management are of great concern to orthopedic surgeons. Although there are several protocols available to deal with these aspects, they are fraught with complications, such as cartilage damage, cardiovascular and neurological intoxication, and systemic adverse responses. Therefore, it is necessary to develop safe and effective perioperative protocols. In the current study, antimicrobial agents/analgesics/growth factor-embedded biodegradable hybrid fixators (polycaprolactone fixator + poly[lactide-coglycolide] sheath-core structured nanofibers) for bone fracture repair were designed.

Methods: The biodegradable hybrid fixators were fabricated using solution-extrusion threedimensional printing and electrospinning. In vitro, the characteristics of the hybrid fixators were examined. Additionally, the release of the incorporated vancomycin, ceftazidime, lidocaine, and bone morphogenetic protein-2 (BMP-2) was evaluated. The in vivo efficacy including drug-eluting properties, fracture repair, and pain management of the biomoleculeloaded nanofibrous fixators was investigated in rabbit rib-fracture models.

Results: The nanofibrous fixators released vancomycin, ceftazidime, and lidocaine in a sustained manner under both in vitro and in vivo conditions and protected BMP-2 from burst release. The implantation of these hybrid fixators around the fractured rib significantly improved animal activities and bone union, indicating that the inclusion of analgesic in the fixator effectively reduced postsurgical pain and thereby helped in recovery.

Conclusion: The novel biomolecule-loaded nanofibrous hybrid fixators resulted in excellent therapeutic outcomes. These fixators may be effective in the repair of rib fractures in clinical settings and may help mitigate surgical complications, such as infection, nonunion, and intolerable postoperative pain.

Keywords: sheath-core structured nanofiber, bone union, biodegradable fixator, antimicrobial, analgesics, growth factor

\section{Introduction}

Technological advancements in medical sciences have accelerated the development of osteosynthesis models and devices. For example, the bone-healing process is no longer reliant on endogenous growth factors alone; instead, synthetic growth factors are added to the fracture site to facilitate fracture repair. ${ }^{1,2}$ In this case, although a high rate of bone union can be achieved, there are several factors that may cause nonunion, including bone loss and defects, excessive periosteal damage during osteosynthesis, and locally colonized microorganisms. ${ }^{3-5}$ Among these, microorganism colonization - infection - before and after osteosynthesis is a dominant factor. ${ }^{6-8}$ 
Surgeons are concerned about perioperative infection, which may lead to missed surgical goals, impaired patient's functions, and increased complications, including fatalities. In orthopedic surgery, bone infection (ie, osteomyelitis) a troublesome complication. ${ }^{9}$ Nonunion is generally observed after osteosynthesis in infected bones and requires long-term parenteral antimicrobial treatment and revision osteosynthesis with new bone transplantation. Thus, the addition of exogenous growth factors may be needed to achieve fracture union and regain normal function.

In contrast, patients undergoing orthopedic surgery are concerned about perioperative pain management. Pain is an important factor in recovery because it has a substantial physical and mental toll on the patients. ${ }^{10,11}$ To counter these problems, several pain management protocols have been proposed. ${ }^{12-14}$ One of these protocols involves soaking surgical tissues with analgesics. ${ }^{15-19}$ Although this local bulk-absorption method is effective, it is accompanied by complications, such as cartilage damage, ${ }^{20}$ cardiovascular intoxication, and impaired neurological responses. $^{21,22}$ Therefore, a safe and effective perioperative pain management protocol is essential.

To address these issues, we developed degradable nanofibrous fixators loaded with antimicrobial agents, analgesics, and growth factors for repairing bone fractures. The fixators were fabricated using three-dimensional (3D) printing and electrospinning/co-electrospinning. In our previous study, ${ }^{23}$ we employed micro-injection molding to prepare biodegradable fixators. Compared with injection molding, 3D printing enables faster and customized product development. ${ }^{24,25}$ The lead time for manufacturing can also be reduced substantially; thus the new designs have shorter time-to-market and can meet customer demands more quickly. Moreover, the electrospinning technique yields nonwoven mats with a thickness in the order of a few nanometers with large surface areas, ease of functionalization, and superior mechanical properties. ${ }^{26,27}$ Furthermore, the co-electrospinning technique can be used to prepare sheath-core structured nanofibers.

We selected poly( $\varepsilon$-caprolactone) (PCL), a popular long-term implantable biomaterial, ${ }^{23,28,29}$ as the backbone of biodegradable fixators because this material undergoes gradual degradation via the hydrolysis of ester linkages under normal physiological conditions (such as those in the human body). To prepare nanofibers, we chose poly (lactic-co-glycolic acid) (PLGA), which is a US Food and Drug Administration-approved copolymer often used in therapeutic devices owing to its excellent biodegradability and biocompatibility. ${ }^{30,31}$

In the current study, we aimed to evaluate the eluting profiles and clinical effectiveness of hybrid fixators with a PCL backbone and biomolecule (antimicrobial medication, analgesic agent, and growth factor)-embedded PLGA nanofibers in a rabbit rib-fracture model. We hypothesized that by implanting these hybrid fixators in the fracture models, bone union could be secured, and the loaded drugs could be eluted, gradually resulting in efficient bone union and growth.

\section{Materials and Methods Materials}

The backbone of the fixators consisted of PCL (molecular weight $[\mathrm{Mn}] \sim 80,000 \mathrm{Da}$ ), and the drug carrier was PLGA, which consisted of lactide and glycolide at a 1:1 ratio $(\mathrm{Mn}$ 33,000 Da). The drugs used were commercial-grade vancomycin hydrochloride, ceftazidime hydrate, and lidocaine hydrochloride. Recombinant human bone morphogenetic protein-2 (BMP-2) was used as the growth factor. The solvents used were dichloromethane (DCM) and 1,1,1,3,3,3-hexafluoro-2-propanol (HFIP). All these chemicals were purchased from Sigma-Aldrich (St. Louis, MO, USA).

\section{Design and Fabrication of Biodegradable Fixators}

Three types of self-locking biodegradable fixators were designed $(\mathrm{H} 2, \mathrm{H} 3$, and $\mathrm{X}$ ), as shown in Figure 1A. The fixators were fabricated on a lab-developed solutionextrusion 3D printer, $^{32}$ which included a solutionextrusion feeder, driving stepper motors and related components, a power source, a syringe controlled by a user interface, an accumulation platform, and a dispensing tip with an outlet of inner diameter $0.18 \mathrm{~mm}$ (Figure 1B). An open Cura interface (Ultimaker B.V., Geldermalsen, The Netherlands) was used to manage the entire 3D printing procedure (Figure 1C).

To print the fixators, $2.5 \mathrm{~g}$ of PCL was mixed with $3.5 \mathrm{~mL}$ of DCM on a magnetic stirrer for $6 \mathrm{~h}$. The mixed solution was then fed into the feeding system of the printer, which consisted of a syringe and plastic dispensing tip. The dispensing tip was moved using a microprocessorcontrolled servo motor during the $3 \mathrm{D}$ printing process, and the PCL solution was extruded out of the tip and deposited on the collection platform. Upon solvent evaporation, 


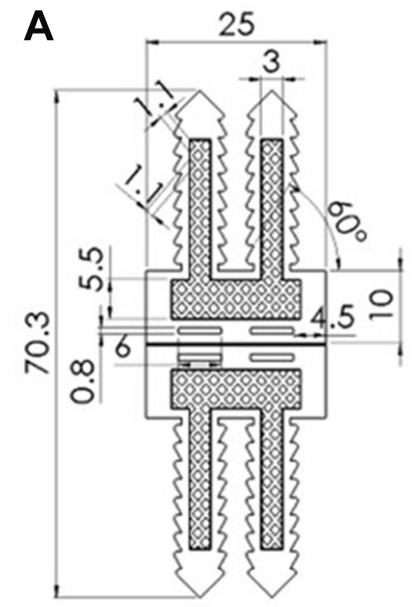

H2 Type

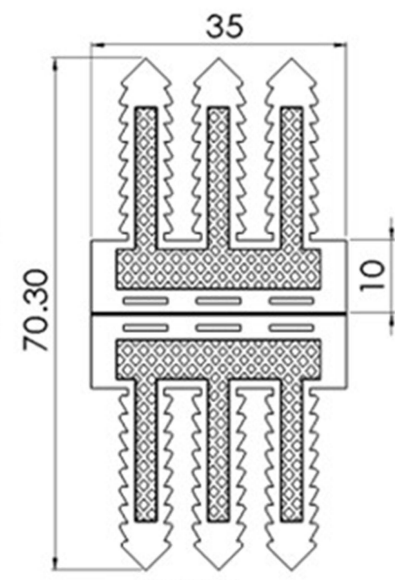

H3 Type

Unit: $\mathrm{mm}$
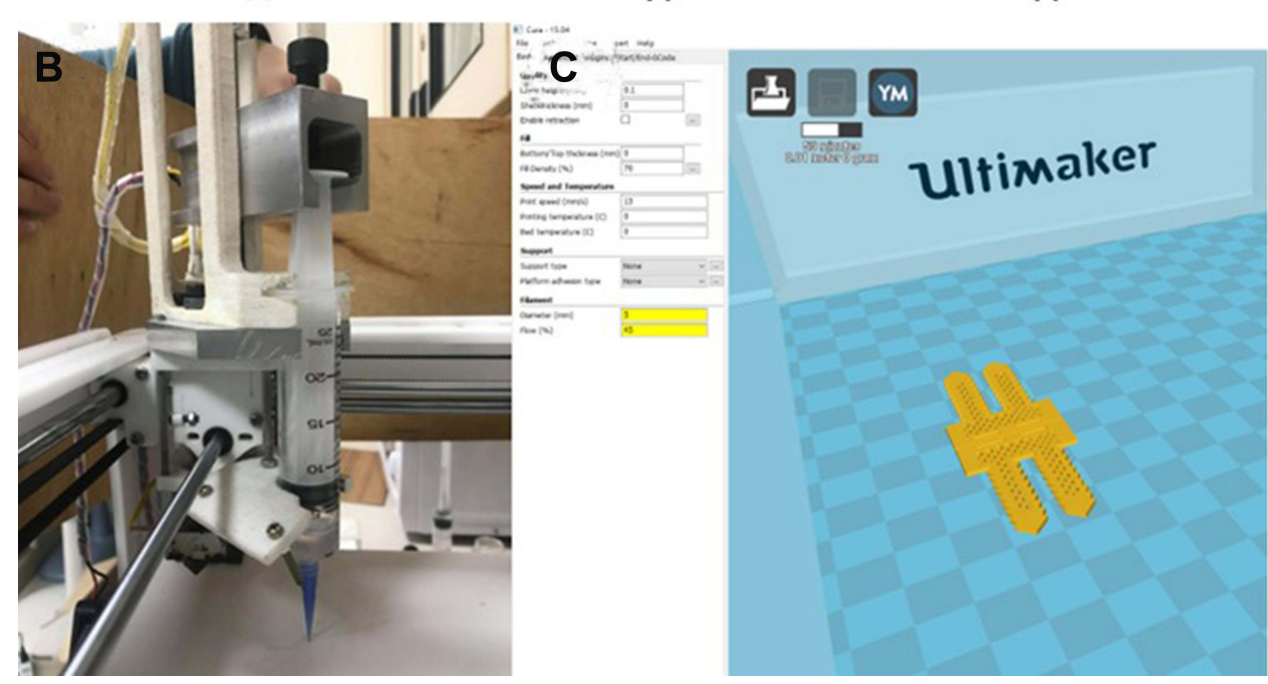

Figure I Fabrication process of the 3D printing process. (A) Layouts and dimensions of the biodegradable fixators. (B) Photograph of the solution-extrusion 3D printer. (C) Cura code used to print the fixators.

biodegradable fixators of different geometrical designs and thicknesses $(0.2$ and $0.4 \mathrm{~mm})$ were obtained.

\section{Mechanical Properties of the Biodegradable Fixators}

A pilot in vitro study was initially conducted to assess the mechanical properties of the biodegradable fixators before in vivo animal studies. Four 6-month-old New Zealand white rabbits weighing 2800-3200 g were used in the biomechanical tests. Each rabbit was placed in an enclosed chamber and subjected to inhalation euthanasia using carbon dioxide ( $2 \mathrm{~L} / \mathrm{min}$ for $3 \mathrm{~min}$ ). Carbon dioxide was pumped for an additional $2 \mathrm{~min}$ to ensure a complete lack of respiration and faded eye color. All animal procedures were approved by the Institutional Animal Care and
Use Committee at the Animal Center of Chang Gung University (CGU 14-102), in accordance with the regulations and guidelines of the Ministry of Health and Welfare, Taiwan.

In total, 21 ribs were harvested (bilateral 5th-7th ribs in each rabbit) and examined. The ribs were prepared by removing the attached muscles and periosteum after euthanasia. Each fixator was fixed to the ribs by passing belts through matching holes and wrapping around the fracture site; the time required for fixing was recorded. Subsequently, the control ribs and fixator-rib assemblies were analyzed using three-point bending on a mechanical testing device (LRX; Lloyd Instruments, Bognor Regis, UK). The specimens were placed on two supporting pins $25 \mathrm{~mm}$ apart from each other, and the loading pin was moved at a speed of $1 \mathrm{~mm} / \mathrm{s}$ in the downward direction until the specimen 
fractured. The applied force and strain on the ribs (control and fixator) during the loading process were recorded.

\section{Fabrication of Biomolecule-Loaded Nanofibers}

Bilayered biomolecule-loaded nanofibers, that is, a regular nanofibrous layer and a sheath-core structured layer, were prepared by electrospinning and co-electrospinning. An electrospinning setup, consisting of a high-voltage supply generating positive direct current voltage (maximal power: $35 \mathrm{kV})$ and current $(4.16 \mathrm{~mA} / 125 \mathrm{~W})$, aluminum sheet, ground electrode, and syringe with a needle $(0.4 \mathrm{~mm}$ internal diameter), was used to fabricate the regular nanofibers. To fabricate these nanofibers, PLGA (1120 mg), vancomycin hydrochloride $(93.3 \mathrm{mg})$, ceftazidime hydrate $(93.3 \mathrm{mg})$, and lidocaine hydrochloride $(93.3 \mathrm{mg}$ ) were mixed with $5 \mathrm{~mL}$ of HFIP and used to fill the syringe pump. This solution was extruded from the syringe at a rate of $0.8 \mathrm{~mL} / \mathrm{h}$ and electrospun on the aluminum sheet, which acted as the collector. The distance from the needle tip to ground electrode was $15 \mathrm{~cm}$, and an $18 \mathrm{kV}$ positive voltage was applied to the polymer solution.

Additionally, to fabricate the sheath-core structured nanofibers, a special coaxial device that concurrently deposited two solutions on the aluminum sheet was used. ${ }^{5}$ PLGA $(840 \mathrm{mg}$ ) was dissolved in $1 \mathrm{~mL}$ of HFIP and applied as the sheath solution, whereas the core solution consisted of $20 \mu \mathrm{g}$ of BMP-2 and $1 \mu \mathrm{L}$ of bovine serum albumin in $1 \mathrm{~mL}$ of phosphate-buffered saline (PBS). For coaxial electrospinning, the PLGA and core liquids were placed in two separate feeding tubes equipped with needles. During co-electrospinning, the liquids were conveyed and spun onto the aluminum sheet at rates of 0.9 and $0.3 \mathrm{~mL} / \mathrm{h}$ for the shell PLGA and core BMP-2 solutions, respectively, using two independently controlled pumps. A $17-\mathrm{kV}$ positive voltage was applied to the solutions, and the distance between the needle tip and ground electrode was maintained at $15 \mathrm{~cm}$.

All electrospinning experiments were carried out at room temperature $\left(25^{\circ} \mathrm{C}\right)$, and the manufactured nanofibrous membranes were heated in a vacuum oven at $40^{\circ} \mathrm{C}$ for $72 \mathrm{~h}$ to vaporize any remaining solvents.

\section{Scanning Electron Microscopy (SEM)}

The morphology of the electrospun nanofibers was evaluated by SEM (JSM7500F; Jeol, Tokyo, Japan). To enhance sample conductivity, before testing, the membrane samples were initially coated with a thin layer of gold. The diameter of 100 randomly selected fibers $(n=3)$ was analyzed using ImageJ software (National Institutes of Health, Bethesda, MD, USA).

\section{Transmission Electron Microscopy (TEM)}

The structure of the sheath-core nanofibers was analyzed by TEM (JEM-2000EXII; Jeol). Samples for TEM analysis were prepared by directly depositing the fibers onto copper grids.

\section{Water Contact Angle}

The hydrophilicity of the electrospun nanofibers was examined in terms of their water contact angles (First Ten Angstroms, Newark, CA, USA). Nanofibrous membrane samples $\left(10 \times 10 \mathrm{~mm}^{2}\right)$ were prepared, and distilled water was slowly dropped onto their surfaces; the contact angles between water droplets and the membranes $(n=3)$ were evaluated using a video monitor. The water contact angle of PCL fixators was also measured for comparison.

\section{Fourier-Transform Infrared (FTIR) Spectroscopy}

To analyze the functional groups present in the synthesized samples, the FTIR spectra of pure PLGA and drugembedded PLGA nanofibers (in the form of pressed $\mathrm{KBr}$ disks) were recorded on a Nicolet iS5 spectrometer (Thermo Fisher Scientific, Waltham, MA, USA) at a resolution of $4 \mathrm{~cm}^{-1}$ over 32 scans in the wave number range of $400-4000 \mathrm{~cm}^{-1}$.

\section{In vitro Release of Pharmaceuticals and BMP-2}

An in vitro elution method was employed to characterize the release patterns of vancomycin, ceftazidime, lidocaine, and BMP-2 from biomolecule-loaded PLGA nanofibers. Nanofiber samples $\left(2 \times 3 \mathrm{~cm}^{2}, 216-220 \mathrm{mg}\right)$ were cut from the electrospun membranes and incubated in $1 \mathrm{~mL}$ of a PBS dissolution medium $(0.15 \mathrm{M}, \mathrm{pH} 7.4)$ at $37^{\circ} \mathrm{C}$ for 24 h. Subsequently, the dissolution medium was isolated and analyzed at $24-\mathrm{h}$ intervals. PBS $(1 \mathrm{~mL})$ was replaced every $24 \mathrm{~h}$ until the sample was fully dissolved. Drug concentrations in the eluents were evaluated using the Hitachi L-2200R multisolvent delivery system (Tokyo, Japan), whereas the BMP-2 level was analyzed using enzyme-linked immunosorbent assay (R\&D Systems, Inc., Minneapolis, MN, USA). 


\section{Rib-Fracture Model}

Twelve 6-month-old New Zealand male rabbits weighing $3.0 \pm 0.2 \mathrm{~kg}$ were used for in vivo experiments. The right sixth rib was selected as the target rib. Each rabbit was anesthetized by inhalation of isoflurane after preoxygenation for $5 \mathrm{~min}$, and the anesthetic was circulated throughout the experimental process. After administering the anesthetic, each rabbit was placed in the decubitus position with the surgical field upward. The skin was prepared and disinfected according to standard antiseptic procedures. A $3-\mathrm{cm}$ incision was made along the rib, and the target rib was reached after dissecting the soft tissue layer and leaving the periosteum undamaged; a short oblique osteotomy was performed on each rib. These rabbits were then randomly divided into control, fixator, and fixator/drug groups ( $n=4 /$ group). Osteosynthesis was not performed in the control group, whereas in the remaining two groups, the osteotomized ribs were fixed either with the fixator alone or with the selected fixator accompanied by biomolecule-loaded PLGA nanofibers, which were circumferentially wrapped around the target rib (Figure 2). Following the surgical procedure, the wound was repaired in layers with absorbable sutures. Postoperatively, the surgical wound was smeared with an antibiotic ointment, and the rabbits were returned to their cages after complete recovery from anesthesia and surgery.

\section{In vivo Release of Drugs and BMP-2 from the Electrospun Nanofibers}

To evaluate the local release and systemic diffusion of antimicrobial agents, analgesic, and growth factors embedded in PLGA nanofibers, the blood and muscle tissue surrounding the implanted PLGA nanofibers were obtained at regular intervals (postoperative days 2, 7, 14, and 28). The measurements were conducted in triplicates $(\mathrm{N}=3)$. After anesthetizing the rabbits, as described above, $1 \mathrm{~mL}$ of blood was extracted from the central auricular artery in their ears. Muscle tissue was obtained through the same skin incision on the operated chest wall. Both samples were preserved in $10 \%$ formalin solution and later analyzed by high-performance liquid chromatography (Hitachi L-2200; Multisolvent Delivery System).

\section{Animal Bioactivity}

We assembled an animal bioactivity cage $(\mathrm{ABC} ; 30 \times$ $30 \times 30 \mathrm{~cm}^{3}$ ) and divided it into nine lattice zones (numbered 1-9). Rabbit movement in these lattice zones was spontaneously detected using electric sensors placed above each lattice and connected to a recording computer. Food and water were supplied in lattice 1 and refilled every 24 h. The rabbits were placed in this designed cage after recovering from anesthesia and surgery for a 7-day observation and pain evaluation period.

During the evaluation process, each $\mathrm{ABC}$ was placed in an isolated room without human interference,

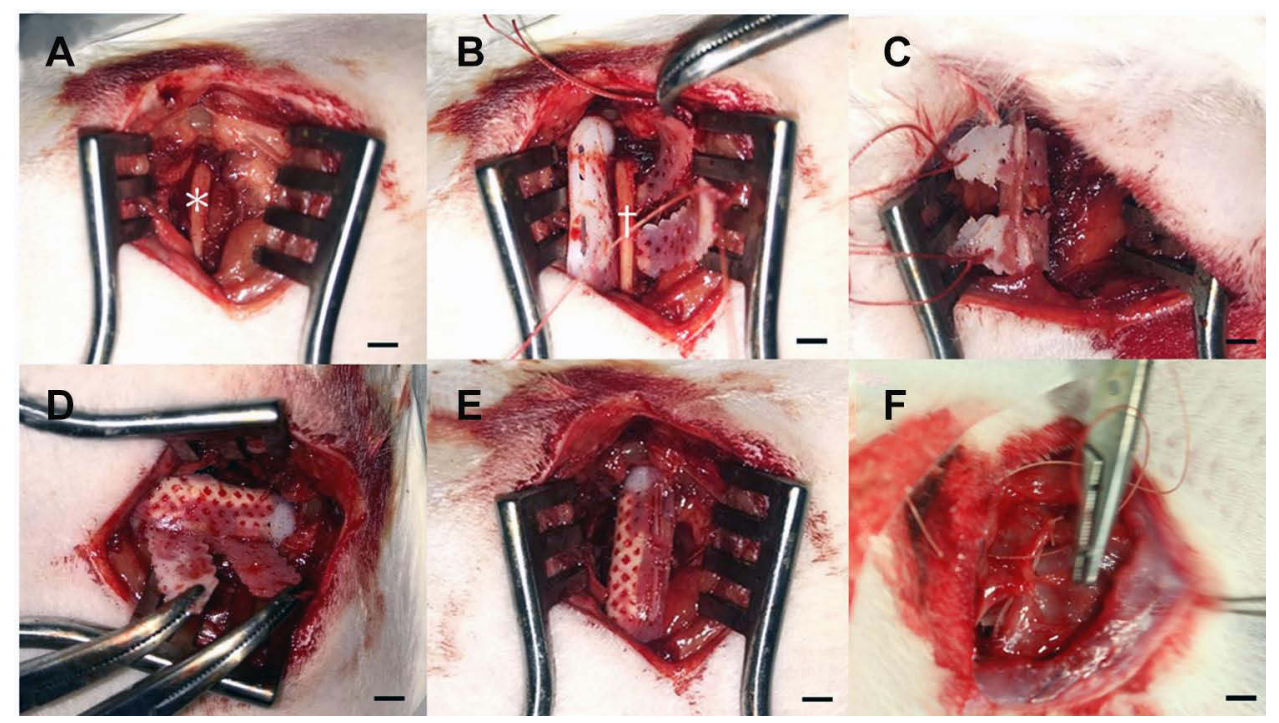

Figure 2 Rib-fracture model and implantation process. (A) The target rib $(*)$ was reached after careful dissection of the soft tissue layer by layer. (B) An oblique fracture was created $(\dagger)$, and the hybrid fixator was placed underneath the osteotomized rib. (C) and (D) Belts were passed through matching holes and tightened. (E) The over-length belts were cut to smoothen the edges of the fixator. (F) The overlying muscular and skin tissues were approximated layer by layer. Scale bar: I cm. 
maintained under the following conditions: constant temperature, $21^{\circ} \mathrm{C}-24^{\circ} \mathrm{C}$; pressure, 1 atmosphere; and humidity, $45 \%-70 \%$. The evaluation was completed after a 7 -day bioactivity-evaluation course; thereafter, the rabbits were moved to regular cages for routine care.

\section{Microcomputed Tomography (Micro-CT) and Histology Evaluation}

Micro-CT images (nanoScan SPECT/CT; Mediso, Hungary) were acquired to monitor the healing of the fractured ribs in the fixator/drug group. The osteotomized rib specimens were excised from rabbits euthanized 28 days after osteosynthesis. The specimens were subjected to histological examination after hematoxylin and eosin staining.

\section{Statistical Analyses}

All statistical data were processed using SPSS software (V17.0 for Windows; SPSS Inc, Chicago, IL, USA). Descriptive statistics are displayed as mean \pm standard deviation. Data were analyzed using the one-way analysis of variance and statistical differences were calculated. The post-hoc Bonferroni method was used to compare pairs of groups to identify significant differences. The observed differences were considered statistically significant at $p<0.05$.

\section{Results}

\section{Mechanical Strength of the Degradable Fixators}

The load-displacement curves of the H2, H3, and $\mathrm{X}$ biodegradable fixators fabricated in this study are shown in Figure 3. Thicker fixators $(0.4 \mathrm{~mm})$ showed significantly higher strengths than their thinner counterparts $(0.2 \mathrm{~mm}) . \mathrm{X}$ and $\mathrm{H} 3$ fixator-repaired ribs were stronger than those repaired with $\mathrm{H} 2$; however, the strength of the repaired ribs was still lower than that of the control (unfractured) ribs. There were no statistical differences among the three fixators $(\mathrm{H} 2$ versus $\mathrm{H} 3, p=$ 0.11 ; $\mathrm{H} 2$ versus $\mathrm{X}, p=0.06$; $\mathrm{H} 3$ versus $\mathrm{X}, p=0.06$ ). Regarding the time required for fixation, $\mathrm{H} 2$ required the least amount of time (H2: $60 \mathrm{~s}$; H3: $90 \mathrm{~s}$; X: $180 \mathrm{~s})$. Therefore, we selected the $\mathrm{H} 2$ fixator for the subsequent in vivo studies.

\section{Characterization of Biomolecule-Loaded Nanofibers}

The SEM images of pure PLGA, drug-loaded, and BMP-2-incorporated nanofibers are shown in

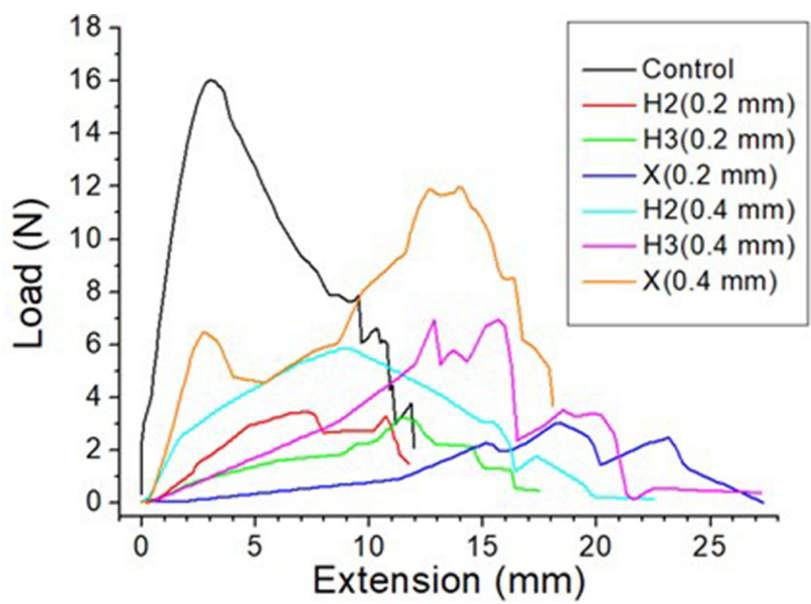

Figure 3 Representative tensile properties of the biodegradable fixators (control represents unfractured ribs).

Figure $4 \mathrm{~A}-\mathrm{C}$, along with their size distributions. The diameter of pure PLGA nanofibers $(577 \pm 465 \mathrm{~nm}$; Figure 4A) was larger than that of drug-embedded PLGA nanofibers $(131 \pm 58 \mathrm{~nm}$; Figure 4B). In the electrospinning procedure, the polymeric solution is extended by the external electric force. With the addition of drugs, the polymer content in the fibers decreased and the nanofibers could be easily stretched by the external force. The sizes of the electrospun fibers decreased accordingly. Meanwhile, Figure 4D shows the TEM image of PLGA/BMP-2 nanofibers in which a sheath-core structure could be clearly observed.

The water contact angles of pure PLGA, drug-loaded, and sheath-core PLGA/BMP-2 nanofibers and PCL fixators were $128.1^{\circ}, 79.2^{\circ}, 121.5^{\circ}$, and $103.5^{\circ}$, respectively. Clearly, the addition of drugs to the nanofibers considerably increased their hydrophilicity (Figure 5).

The FTIR spectra of pure PLGA and drug-loaded PLGA electrospun mats are shown in Figure 6. The new vibration peak at $1676 \mathrm{~cm}^{-1}$ in the spectrum corresponding to drug-loaded PLGA was attributed to the $\mathrm{C}=\mathrm{C}$ bonds of the aromatic rings in the drug molecules. The peak at $1762 \mathrm{~cm}^{-1}$ was ascribed to the $\mathrm{C}=\mathrm{O}$ bonds of the incorporated drugs. The peak near $2996 \mathrm{~cm}^{-1}$, corresponding to $\mathrm{CH}_{2}$ bonds, was enhanced upon the addition of drugs to the nanofiber mat. Moreover, the absorption band observed at $3422 \mathrm{~cm}^{-1}$ in the FTIR spectrum of drug-loaded PLGA could be attributed to the N-H bonds of vancomycin, ceftazidime, and lidocaine. Thus, the obtained FTIR spectra demonstrated the successful incorporation of antimicrobial and analgesic agents in PLGA nanofibers. 

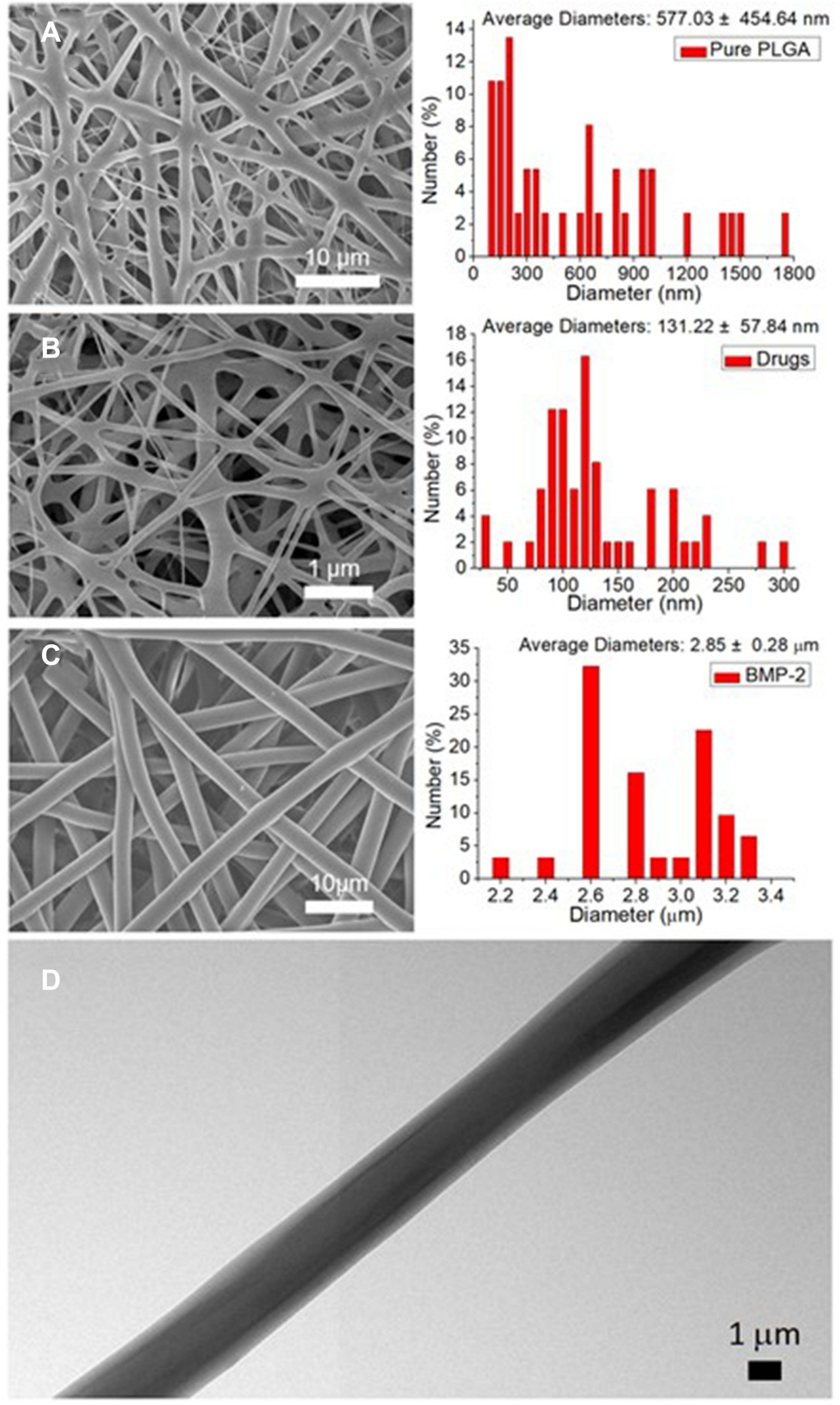

Figure 4 Scanning electron microscopy images of pure and biomolecule-loaded poly(lactic-co-glycolic acid) (PLGA) nanofibers and their size distributions. (A) Pure PLGA nanofibers, (B) drug-loaded nanofibers, and (C) bone morphogenetic protein-2 (BMP-2)-incorporated sheath-core structured nanofibers. (D) Transmission electron microscopy image of BMP-2-incorporated sheath-core structured nanofibers. 


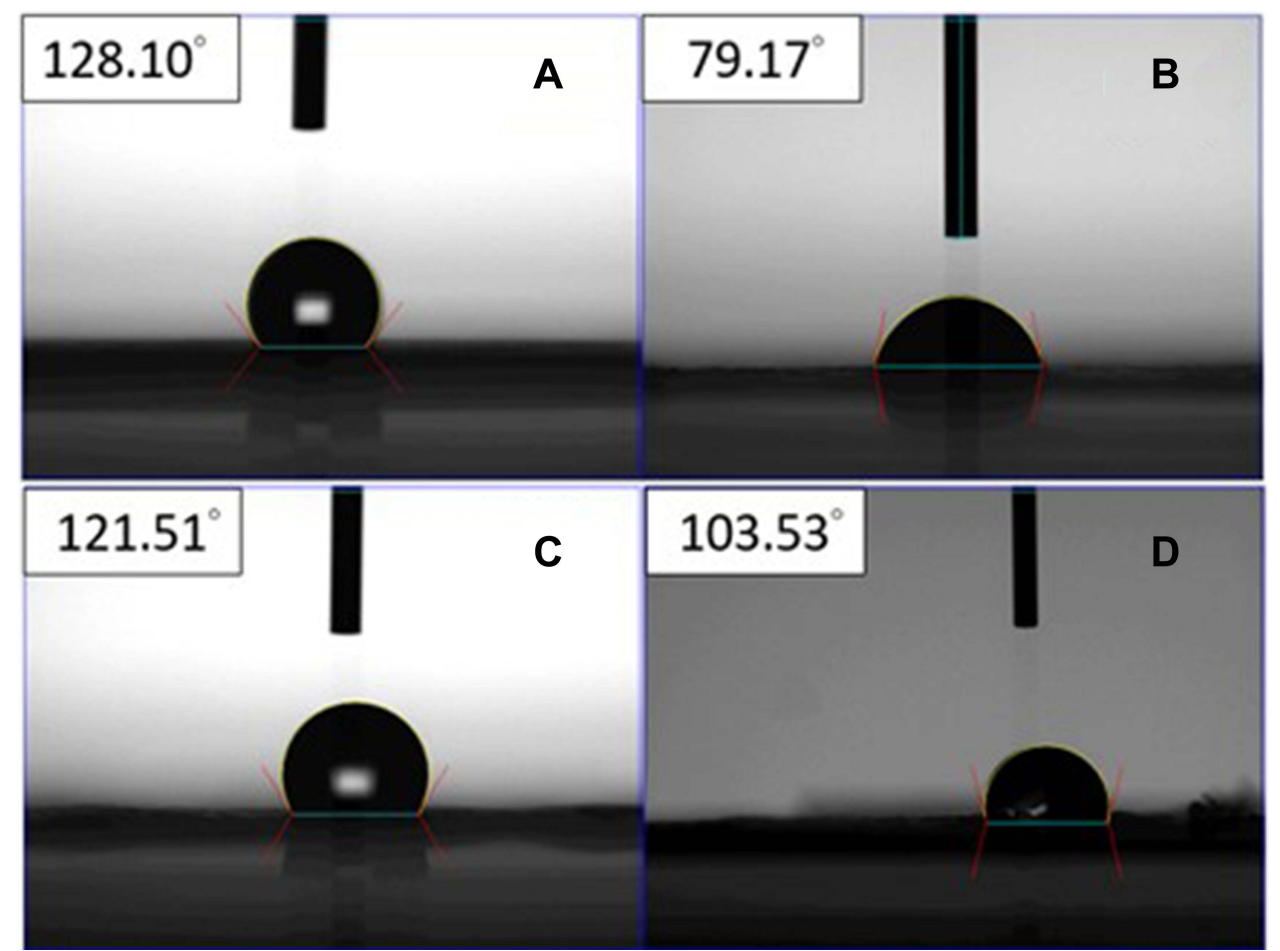

Figure 5 Water contact angles of (A) pure PLGA nanofibers, (B) drug-loaded PLGA nanofibers, (C) sheath-core structured BMP-2/PLGA nanofibers, and (D) H2 fixator.

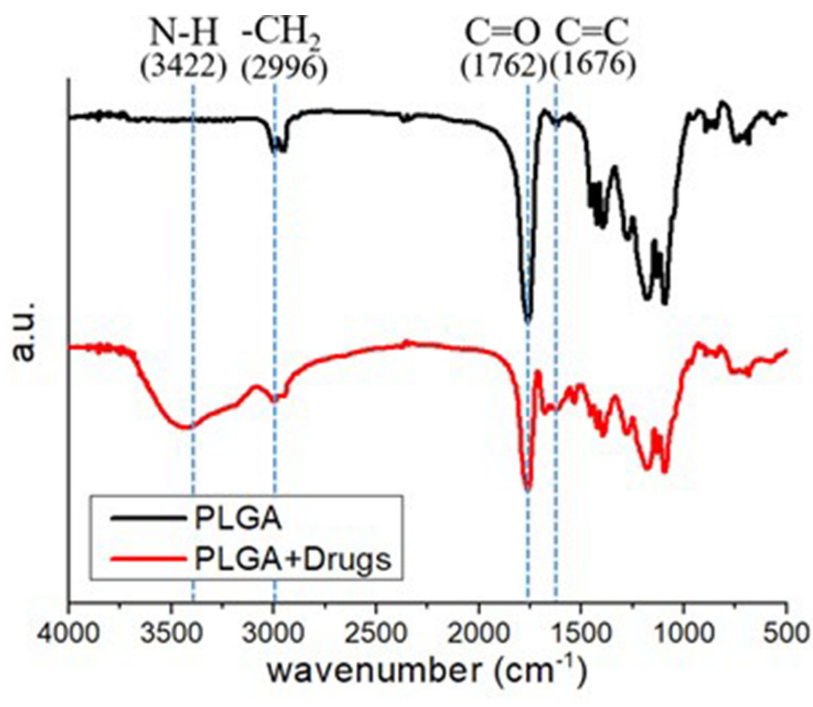

Figure 6 Fourier-transform infrared spectroscopy spectra of pure and drug-loaded poly(lactic-co-glycolic acid) (PLGA) nanofibers.

\section{In vitro and in vivo Elution Profiles}

The in vitro elution profiles of drugs loaded in the PLGA nanofibers are shown in Figure $7 \mathrm{~A}$ and $\mathrm{B}$. The results suggested that the drug-containing nanofibers released vancomycin and ceftazidime in a sustained manner at concentrations higher than their minimum inhibitory concentrations (MICs) for 30 and 24 days, respectively.
Additionally, lidocaine was released for over 15 days. In all three cases, a high rate of elution was observed during the first 3 days, followed by gradual and continued elution, except that a second peak of drug release was found. The in vitro elution of BMP-2 from the nanofibers was also characterized. As shown in Figure 7C, the sheath-core structured nanofibers could sustainably release BMP-2 for more than 30 days in a fairly stable manner. This may be because BMP-2 was embedded in the core part of the sheath-core nanofibers. In other words, the PLGA sheath exerted a shielding effect and protected BMP-2 from a burst release. The in vivo concentrations of drugs released in the animal models were also measured (Figure 7D). Importantly, the nanofibers released the drugs in an extended manner for more than 28 days. Moreover, although the drug concentrations in the tissues were high, drug levels in the blood remained low throughout the study.

\section{Efficacy of Pain Control of Drug-Loaded PLGA Nanofibers in Animal Models}

To determine the efficacy of the incorporated analgesics, animal activities were examined post surgery. Figure 8A shows the numbers of lattice zones (1-9) of the ABC. The data in Figure $8 \mathrm{~B}$ show that lattice zone 1 (diet) was the 

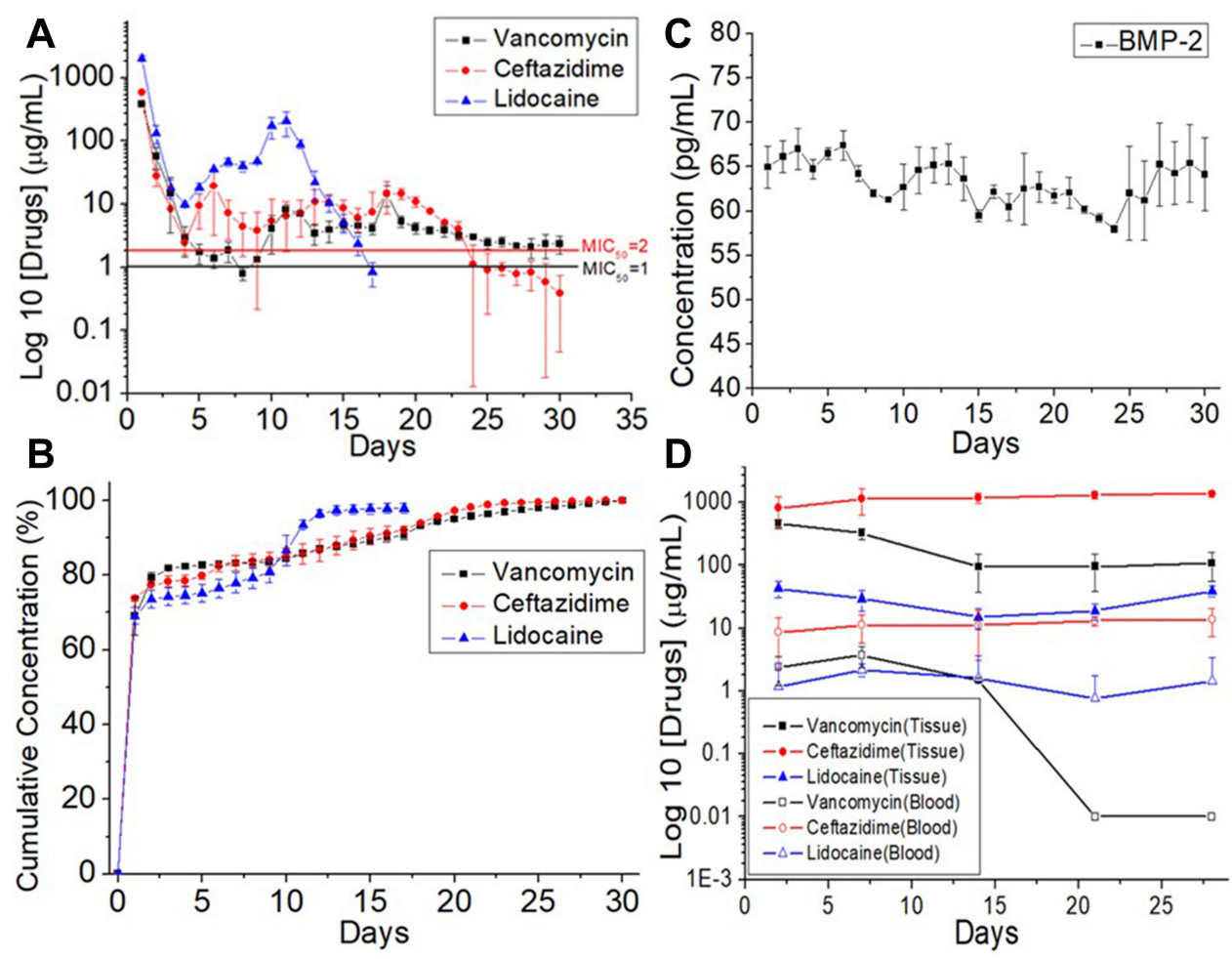

Figure 7 In vitro release profiles of carried pharmaceuticals (A-C). (A) Daily and (B) cumulative release of vancomycin, ceftazidime, and lidocaine from poly(lactic-coglycolic acid) (PLGA) nanofibers. (C) Bone morphogenetic protein-2 (BMP-2) released from PLGA sheath-core nanofibers. (D) In vivo drug release from PLGA nanofibers in tissue and blood. Values represent mean \pm standard deviation $(n=12)$.

most inhabited in the control group, whereas lattice zone 7 (corner) was the most visited zone in the fixator/drug group. Furthermore, lattice zone 5 was the least inhabited zone in both groups. A comparison of daily activity counts (Figure 8C) on each day between the fixator and fixator/ drug groups revealed significant differences $(p<0.05)$. Although there was no significant difference between the control and fixator/drug groups, the 7-day counts indicated significant differences between the fixator and fixator/drug groups $(p<0.05)$.

\section{Gross and Histological Examinations}

Gross and histological examinations of the target ribs treated with hybrid fixators were carried out (Figure 9AD). Gross examination revealed that the PLGA nanofibers were absorbed with the PCL-based rib fixators around the rib without breaking the locking mechanism. Perioperative infection was not observed in any specimen. All fractures showed union without excessive inflammatory process or ectopic bone formation. CT scans of the ribs revealed a continuous and circumferential bridging callus in three out of four specimens. One specimen revealed incomplete union, representing cortical discontinuity (Figure 9B). Histological examination of the tissue surrounding the hybrid fixators indicated rich mono- and multi-nucleated cells, along with a certain number of fibroblasts in the first 2 weeks (Figure 10A and B). By week 3 (Figure 10C), the deeper layers of the membranes consisted of fibroblast-like spindle cells haphazardly oriented in an extracellular matrix-rich stroma. Membranous tissue was rich in small blood vessels with scattered eosinophils and lymphocytes and showed a gradual decrease in tissue reaction by weeks 4 (Figure 10D).

\section{Discussion}

In this study, we fabricated biomolecule (antibiotics, analgesics, and bone growth factor)-loaded nanofibrous biodegradable fixators by $3 \mathrm{D}$ printing and electrospinning/co-electrospinning for promoting bone union in osteosynthesis. The biodegradable fixators described herein could overcome the limitations posed by traditional metallic fixators, such as mechanical load mismatch, graft lacerations, secondary surgical removal, and hindrance during postoperative magnetic resonance imaging and CT scanning. Furthermore, the biomolecule-loaded degradable 


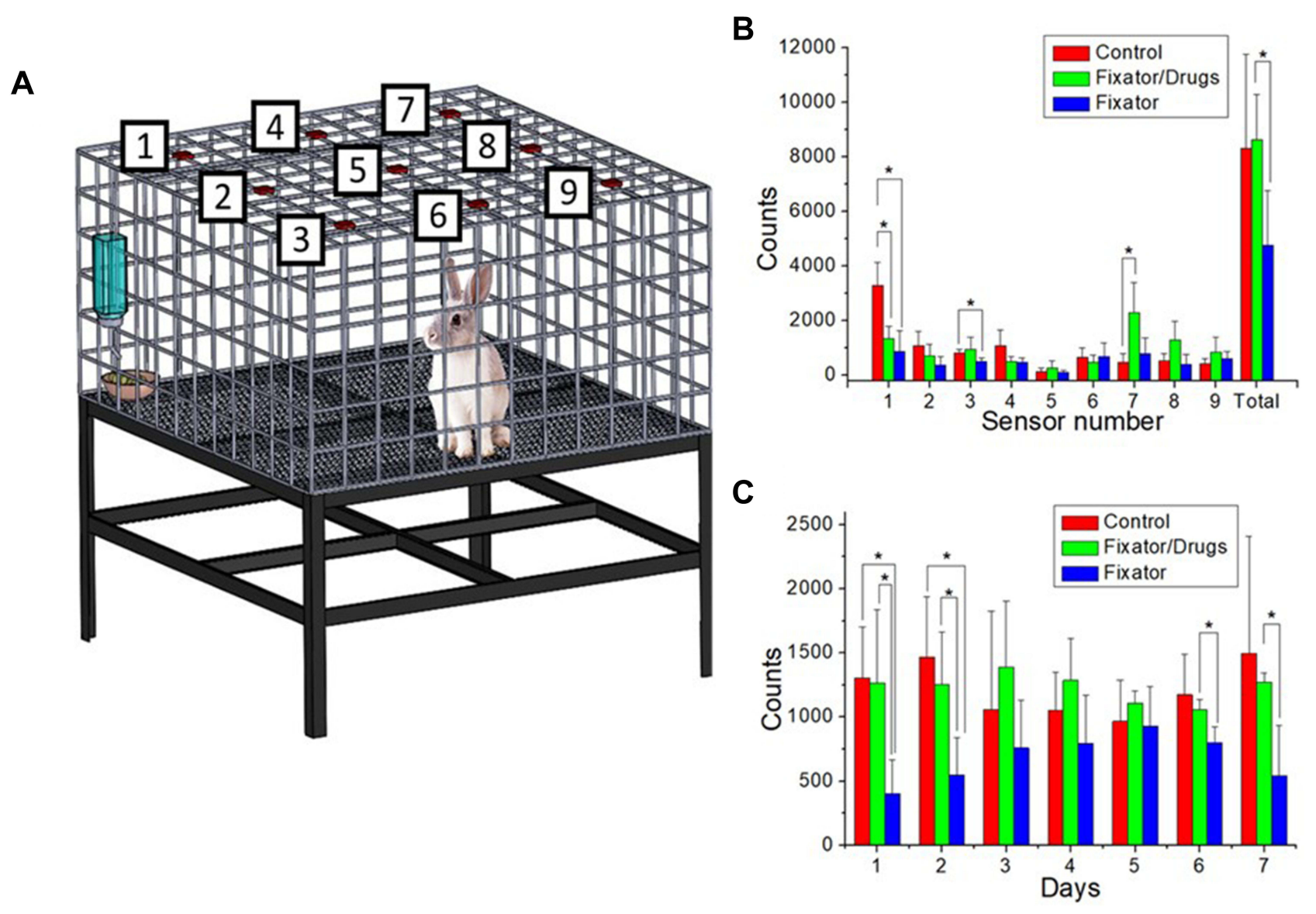

Figure 8 Schematic of an animal bioactivity cage $(A B C)$. (A) Nine different lattice zones contained in the $A B C$ for post-surgery evaluation. Animal activity counts (B) in each $A B C$ lattice zones (sensor number) and $(\mathbf{C})$ on different days for a total of 7 days. Values represent mean \pm standard deviation $(n=12) ;{ }^{*}<<0.05$.

fixators delivered drugs and growth factors in a sustained manner to the target sites to enhance bone healing.

Perioperative infection during osteosynthesis is a concern in orthopedic surgeries. Various prophylactic approaches to control bacterial colonization are used to ensure clean orthopedic surgeries, including prolonged systemic antimicrobial medications, local antimicrobial solution rinse and irrigation, and local application of an antimicrobial powder. ${ }^{33-36}$ However, these methods have their own limitations, such as a less-than-optimal infection prevention, reaction with local tissues, healing inhibition, and systemic toxicity. Other researchers used PMMA as a carrier of antibiotics for the purpose of controlled drug release with satisfactory results. ${ }^{40,41}$ However, the PMMA was not biodegradable and should be surgically removed. In this study, we designed and developed multi-drugloaded fixators using degradable PCL and PLGA to provide an all-in-one approach for infection control, fracture union, and analgesia. Once the drugs are released, the degradable carrier is also resorbed, and no additional surgery is required to remove the carrier. Moreover, previous studies revealed that a locally applied antibioticloaded carrier (biodegradable or non-biodegradable) would not increase the systemic concentration of the drugs. ${ }^{31,42}$ In our study, the concentration of the applied antimicrobial agents remained above the MIC90, yet localized, without systemic spreading. Compared with other delivery agents, the tunable porosity and pore size of nanofibers permit them to mimic the extracellular matrix (ECM) to enhance cell proliferation. The large surface-tovolume ratio of electrospun nanofibers also offers a considerable number of bioactive binding sites, making it a promising candidate for multiple biomedical applications. Owing to these advantages, it is suggested that the hybrid fixator design described in the current study can not only be used during surgeries as an infection prophylaxis but also as a definite treatment for chronic osteomyelitis for complete eradication of microorganisms.

The treatment course described in this study can be especially helpful in cases of chronic osteomyelitis in 


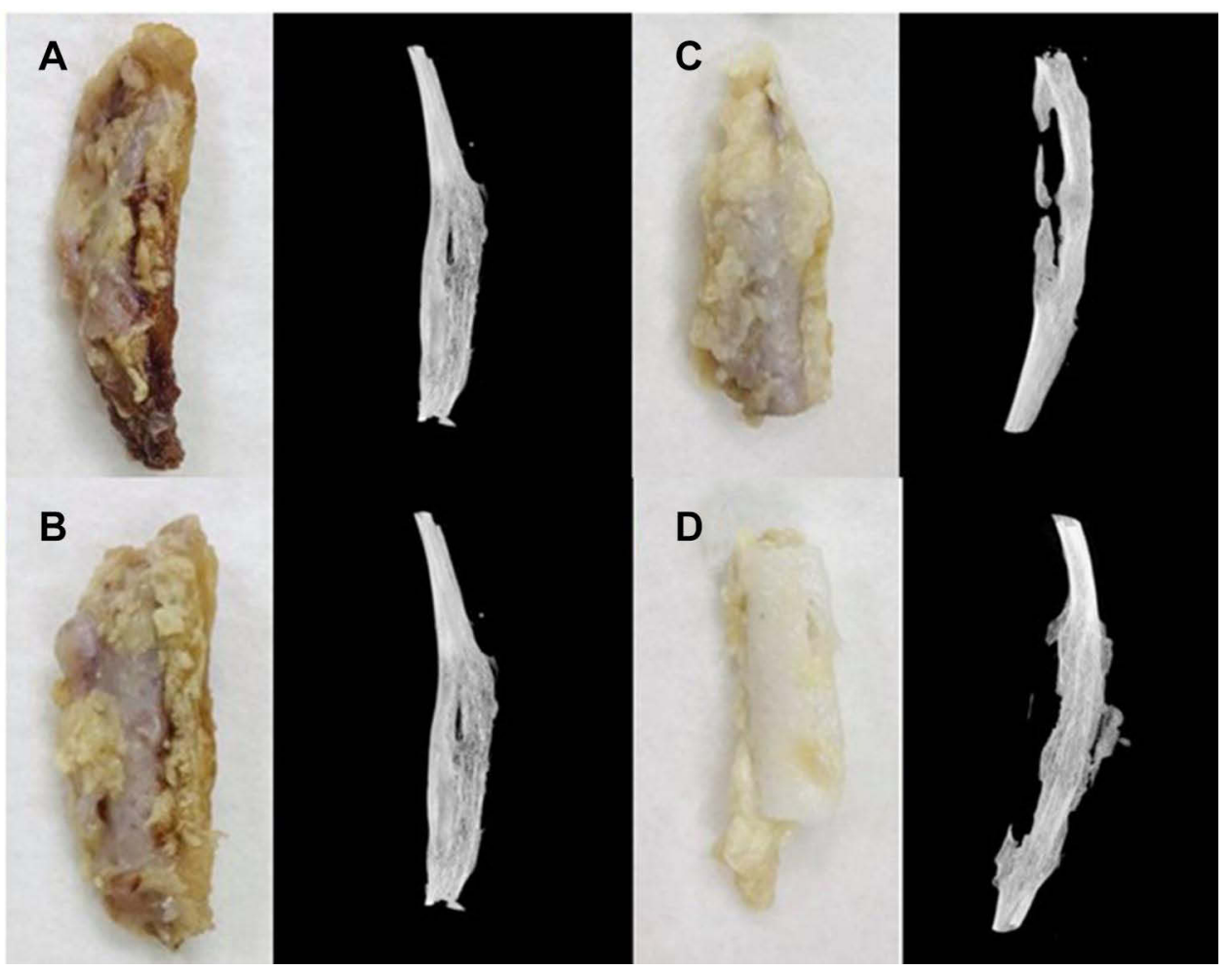

Figure 9 Radiological results of the animal study. (A-D) Experimental specimens (rib-fixator composites) and corresponding three-dimensional microcomputed tomography images of the fixator/drugs group. Scale bar, $1 \mathrm{~cm}$.

fracture nonunion (infective nonunion), for both patients and surgeons. Although we did not conduct in vivo bactericidal assays, the PLGA nanofibers were loaded with two common antimicrobial medications, that is, vancomycin and ceftazidime, which can eradicate most grampositive and gram-negative microorganisms encountered in osteomyelitis. ${ }^{37-39}$ The hybrid fixator assembly described herein exhibited sustained elution of antimicrobial drugs at levels higher than their MIC for up to 28 days, thereby creating an environment in which infection could be effectively prevented and osteomyelitis can be treated. Additionally, the continuous elution of BMP-2 from PLGA nanofibers is expected to facilitate repair in nonunion cases. As observed in our analyses, all fractures in the animal models were united within 28 days without excessive inflammatory reaction or ectopic bone formation.

Another significant finding of our study was the analgesic effect provided by lidocaine eluted from multidrug-loaded PLGA nanofibers. One of the biggest concerns of patients receiving medical treatment is pain, particularly in orthopedic surgeries. Currently, there are several analgesic protocols available for application in the postoperative period. ${ }^{12-19}$ However, inadequate pain control (via oral administration or parietal injection) and systemic adverse effects (from bulky absorption during intra-articular injection) are the drawbacks of these protocols. In this study, an analgesic was embedded in the biodegradable PLGA nanofibers, and its slow and continuous release is expected to greatly relieve postoperative pain. In our studies on the postoperative activities of rabbits that underwent surgery, we found that the animals implanted with analgesic-containing PLGA nanofibers were as active as those in the control group. In fact, the animals in the control and drug-loaded fixator groups were considerably more active than those in the fixator-only group (in terms of both daily activity and 7-day activity). This result demonstrates that the inclusion of analgesic in the fixator was effective in reducing postsurgical pain and thus helped recovery.

Although we endeavored to minimize any bias in our in vitro and in vivo analyses, there still remained some limitations. First, we did not create an infected animal model in which the bactericidal effect of the applied materials could have been evaluated during the study. However, the local concentration of antibiotics was greater than the MIC90 $(2 \mu \mathrm{g} / \mathrm{mL})$, indicating the bactericidal effects. Second, the animal model used was not 


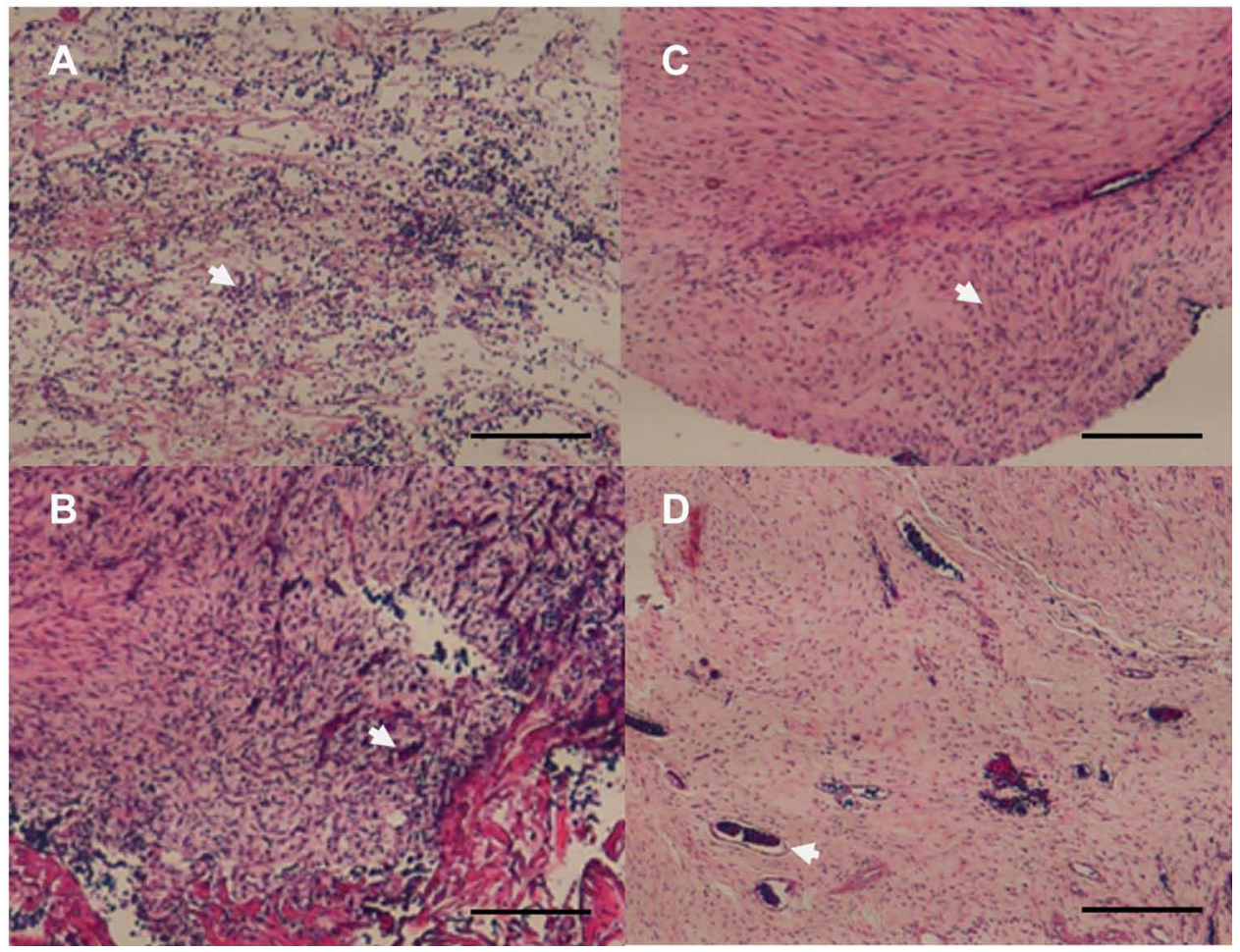

Figure 10 Histological images (hematoxylin and eosin staining) of the fixator/drug group. Rich mono- and multi-nuclear cells (arrow) were observed at I week (A) and 2 weeks (B). Oriented fibroblast-like spindle cells (arrow) could be observed at 3 weeks (C), and several small blood vessels (arrow) were observed at 4 weeks (D) post implantation. Scale bar, $500 \mu \mathrm{m}$.

a nonunion model; hence, the capacity of the hybrid fixator to facilitate bone union could be inferred only from laboratory and image findings. Third, the postoperative behavior of the animals was evaluated only using an ABC. Finally, the relevance of our findings to humans for bone fracture repair remains unclear and needs to be further explored. However, the strength of this study lies in the fact that the novel biomolecule-loaded nanofibrous fixator resulted in an excellent therapeutic outcome. Thus, these devices may be helpful in dealing with surgical complications such as infection, nonunion, and intolerable postoperative pain.

\section{Conclusions}

In this study, we developed vancomycin-, ceftazidime-, lidocaine-, and BMP-2-loaded biodegradable nanofibrous fixators based on PCL and PLGA for bone fracture repair. When tested in animal models, these biodegradable fixators ensured sustained release of vancomycin, ceftazidime, and lidocaine for 30,24, and 15 days, respectively, under in vitro conditions, and for 28 days (each compound) under in vivo conditions. In addition, the fixators released high concentrations of BMP-2 for more than 28 days and promoted bone union. Taken together, we successfully designed and developed a hybrid structure consisting of a PCL-based fixator and PLGA-based nanofibrous membranes loaded with antimicrobial agents, analgesics, and growth factors to promote bone union in fracture cases, aid in countering infections, and ameliorate postoperative pain.

\section{Acknowledgments}

We thank the technical support staff at the molecular imaging facilities of the Laboratory Animal Center, Chang Gung Memorial Hospital-Linkou. This work was supported by the Ministry of Science and Technology, Taiwan [Grant number 109-2221-E-182-058-MY2] and the Chang Gung Memorial Hospital [grant numbers CMRPG3K2341 and CRRPG3K0081].

\section{Disclosure}

The authors declare that they have no competing interests.

\section{References}

1. Schmidmaier G, Wildemann B, Ostapowicz D, et al. Long-term effects of local growth factor (IGF-I and TGF-beta 1) treatment on fracture healing. A safety study for using growth factors. J Orthop Res. 2004;22(3):514-519. doi:10.1016/j.orthres.2003.09.009 
2. Dumic-Cule I, Peric M, Kucko L, et al. Bone morphogenetic proteins in fracture repair. Int Orthop. 2018;42(11):2619-2626. doi:10.1007/ s00264-018-4153-y

3. Bell A, Templeman D, Weinlein JC. Nonunion of the femur and tibia: an update. Orthop Clin. 2016;47(2):365-375. doi:10.1016/j. ocl.2015.09.010

4. Ross KA, O'Halloran K, Castillo RC, et al. Prediction of tibial nonunion at the 6-week time point. Injury. 2018;49(11):2075-2082. doi:10.1016/j.injury.2018.07.033

5. Tian R, Zheng F, Zhao W, et al. Prevalence and influencing factors of nonunion in patients with tibial fracture: systematic review and meta-analysis. J Orthop Surg Res. 2020;15(1):377. doi:10.1186/ s13018-020-01904-2

6. Willey M, Karam M. Impact of infection on fracture fixation. Orthop Clin. 2016;47(2):357-364. doi:10.1016/j.ocl.2015.09.004

7. Raven TF, Moghaddam A, Ermisch C, et al. Use of Masquelet technique in treatment of septic and atrophic fracture nonunion. Injury. 2019;50(Suppl 3):40-54. doi:10.1016/j.injury.2019.06.018

8. Zura R, Mehta S, Della Rocca GJ, et al. Biological risk factors for nonunion of bone fracture. JBJS Rev. 2016;4(1):e5. doi:10.2106/ JBJS.RVW.O.00008

9. Metsemakers WJ, Kortram K, Morgenstern M, et al. Definition of infection after fracture fixation: a systematic review of randomized controlled trials to evaluate current practice. Injury. 2018;49 (3):497-504. doi:10.1016/j.injury.2017.02.010

10. Pitimana-aree S, Visalyaputra S, Komoltri C, et al. An economic evaluation of bupivacaine plus fentanyl versus ropivacaine alone for patient-controlled epidural analgesia after total-knee replacement procedure: a double-blinded randomized study. Reg Anesth Pain Med. 2005;30:446-451. doi:10.1097/00115550-200509000-00005

11. Zaslansky R, Eisenberg E, Peskin B, et al. Early administration of oral morphine to orthopedic patients after surgery. J Opioid Manag. 2006;2:88-92. doi:10.5055/jom.2006.0014

12. Wyatt MC, Wright T, Locker J, et al. Femoral nerve infusion after primary total knee arthroplasty: a prospective, double-blind, randomised and placebo-controlled trial. Bone Joint Res. 2015;4(2):11-16. doi:10.1302/2046-3758.42.2000329

13. Olive DJ, Barriton MJ, Simone SA, et al. A randomised controlled trial comparing three analgesia regimens following total knee joint replacement: continuous femoral nerve block, intrathecal morphine or both. Anaesth Intensive Care. 2015;43(4):454-460. doi:10.1177/ $0310057 \mathrm{X} 1504300406$

14. Peterson JR, Steele JR, Wellman SS, et al. Surgeon-performed high-dose bupivacaine periarticular injection with intra-articular saphenous nerve block is not inferior to adductor canal block in total knee arthroplasty. $J$ Arthroplasty. 2020;35(5):1233-1238. doi:10.1016/j.arth.2020.01.030

15. Chen DW, Hu CC, Chang $\mathrm{YH}$, et al. Intra-articular bupivacaine reduces postoperative pain and meperidine use after total hip arthroplasty: a randomized, double-blind study. $J$ Arthroplasty. 2014;29:2457-2461. doi:10.1016/j.arth.2013.12.021

16. Kendall MC, Alves LJC, De Oliveira G. Liposome bupivacaine compared to plain local anesthetics to reduce postsurgical pain: an updated meta-analysis of randomized controlled trials. Pain Res Treat. 2018;2018:5710169.

17. Berend ME, Berend KR, Lombardi JAV. Advances in pain management: game changers in knee arthroplasty. Bone Joint J. 2014;96-B (96-B):7-9. doi:10.1302/0301-620X.96B11.34514

18. Hyland SJ, Deliberato DG, Fada RA, et al. Liposomal bupivacaine versus standard periarticular injection in total knee arthroplasty with regional anesthesia: a prospective randomized controlled trial. J Arthroplasty. 2019;34(3):488-494. doi:10.1016/j.arth.2018.11.026

19. Ali A, Sundberg M, Hansson U, et al. Doubtful effect of continuous intraarticular analgesia after total knee arthroplasty: a randomized double-blind study of 200 patients. Acta Orthop. 2015;86:373-377. doi:10.3109/17453674.2014.991629
20. Buchko JZ, Gurney-Dunlop T, Shin JJ. Knee chondrolysis by infusion of bupivacaine with epinephrine through an intra-articular pain pump catheter after arthroscopic ACL reconstruction. Am J Sports Med. 2015;43(2):337-344. doi:10.1177/0363546514555667

21. Sekimoto K, Tobe M, Saito S. Local anesthetic toxicity: acute and chronic management. Acute Med Surg. 2017;4(2):152-160. doi:10.1002/ams2.265

22. Ballieul RJ, Jacobs TF, Herregods S, et al. The peri-operative use of intra-articular local anesthetics: a review. Acta Anaesthesiol Belg. 2009;60(2):101-108.

23. Yu YH, Fan CL, Hsu YH, et al. A novel biodegradable polycaprolactone fixator for osteosynthesis surgery of rib fracture: in vitro and in vivo study. Materials. 2015;8(11):7714-7722. doi:10.3390/ ma8115415

24. Kotta S, Nair A, Alsabeelah N. 3D printing technology in drug delivery: recent progress and application. Curr Pharm Des. 2018;24 (42):5039-5048. doi:10.2174/1381612825666181206123828

25. Attaran M. The rise of 3-D printing: the advantages of additive manufacturing over traditional manufacturing. Bus Horiz. 2017;60 (5):677-688. doi:10.1016/j.bushor.2017.05.011

26. Hokmabad VR, Davaran S, Ramazani A, et al. Design and fabrication of porous biodegradable scaffolds: a strategy for tissue engineering. J Biomater Sci Polym Ed. 2017;28(16):1797-1825. doi:10.1080/ 09205063.2017.1354674

27. Agarwal S, Wendorff JH, Greiner A. Use of electrospinning technique for biomedical applications. Polymer. 2008;49(26):5603-5621. doi:10.1016/j.polymer.2008.09.014

28. Kim J, Kudisch M, Mudumba S, et al. Biocompatibility and pharmacokinetic analysis of an intracameral polycaprolactone drug delivery implant for glaucoma. Invest Ophthalmol Vis Sci. 2016;57 (10):4341-4346. doi:10.1167/iovs.16-19585

29. Chou YC, Cheng YS, Hsu YH, et al. Biodegradable nanofiber-membrane for sustainable release of lidocaine at the femoral fracture site as a periosteal block: in vitro and in vivo studies in a rabbit model. Colloids Surf B Biointerfaces. 2016;140:332-341. doi:10.1016/j.colsurfb.2016.01.011

30. Yu YH, Hsu YH, Chou YC, et al. Sustained relief of pain from osteosynthesis surgery of rib fracture by using biodegradable lidocaine-eluting nanofibrous membranes. Nanomedicine. 2016;12 (7):1785-1793. doi:10.1016/j.nano.2016.04.015

31. Yu YH, Lee D, Hsu YH, et al. A three-dimensional printed polycaprolactone scaffold combined with co-axially electrospun vancomycin/ceftazidime/bone morphological protein-2 sheath-core nanofibers for the repair of segmental bone defects during the Masquelet procedure. Int $J$ Nanomed. 2020;15:913-925. doi:10.2147/IJN.S238478

32. Chen JM, Lee D, Yang JW, et al. Solution-extrusion additive manufacturing of biodegradable polycaprolactone. Appl Sci. 2020;10:3189. doi:10.3390/app10093189

33. Conterno LO, Turchi MD. Antibiotics for treating chronic osteomyelitis in adults. Cochrane Database Syst Rev. 2013;2(9):CD004439.

34. Dudareva M, Kümin M, Vach W, et al. Short or long antibiotic regimes in orthopaedics (SOLARIO): a randomised controlled open-label non-inferiority trial of duration of systemic antibiotics in adults with orthopaedic infection treated operatively with local antibiotic therapy. Trials. 2019;20(1):693. doi:10.1186/s13063-019-3832-3

35. Cancienne JM, Burrus MT, Weiss DB, et al. Applications of local antibiotics in orthopedic trauma. Orthop Clin North Am. 2015;46 (4):495-510. doi:10.1016/j.ocl.2015.06.010

36. Hake ME, Young H, Hak DJ, et al. Local antibiotic therapy strategies in orthopaedic trauma: practical tips and tricks and review of the literature. Injury. 2015;46(8):1447-1456. doi:10.1016/j. injury.2015.05.008

37. Inzana JA, Schwarz EM, Kates SL, et al. Biomaterials approaches to treating implant-associated osteomyelitis. Biomaterials. 2016;81:58-71. doi:10.1016/j.biomaterials.2015.12.012 
38. Hsu YH, Hu CC, Hsieh PH, et al. Vancomycin and ceftazidime in bone cement as a potentially effective treatment for knee periprosthetic joint infection. J Bone Joint Surg Am. 2017;99(3):223-231. doi:10.2106/JBJS.16.00290

39. Hsu YH, Chen DW, Tai CD, et al. Biodegradable drug-eluting nanofiber-enveloped implants for sustained release of high bactericidal concentrations of vancomycin and ceftazidime: in vitro and in vivo studies. Int $J$ Nanomed. 2014;9:4347-4355. doi:10.2147/ IJN.S66526

40. Nau C, Seebach C, Trumm A, et al. Alteration of Masquelet's induced membrane characteristics by different kinds of antibiotic enriched bone cement in a critical size defect model in the rat's femur. Injury. 2016;47(2):325-334. doi:10.1016/j.injury.2015.10.079
41. Shah SR, Smith BT, Tatara AM, et al. Effects of local antibiotic delivery from porous space maintainers on infection clearance and induction of an osteogenic membrane in an infected bone defect. Tissue Eng Part A. 2017;23(3-4):91-100. doi:10.1089/ten. tea.2016.0389

42. Foster AL, Boot W, Stenger V, et al. Single-stage revision of MRSA orthopedic device-related infection in sheep with an antibiotic-loaded hydrogel. J Orthop Res. 2021;39(2):438-448. doi:10.1002/jor.24949

\section{Publish your work in this journal}

The International Journal of Nanomedicine is an international, peerreviewed journal focusing on the application of nanotechnology in diagnostics, therapeutics, and drug delivery systems throughout the biomedical field. This journal is indexed on PubMed Central, MedLine, CAS, SciSearch ${ }^{\mathbb{R}}$, Current Contents ${ }^{\mathbb{R}} /$ Clinical Medicine, $^{2}$
Journal Citation Reports/Science Edition, EMBase, Scopus and the Elsevier Bibliographic databases. The manuscript management system is completely online and includes a very quick and fair peer-review system, which is all easy to use. Visit http://www.dovepress.com/ testimonials.php to read real quotes from published authors. 